\title{
Magnetically textured ferrofluid in a non-magnetic matrix: Magnetic properties
}

\author{
MRUDUL GADHVI ${ }^{\dagger}$, R V UPADHYAY*, KINNARI PAREKH and R V MEHTA \\ Department of Physics, Bhavnagar University, Bhavnagar 364 002, India \\ †Physics Department, St. Xavier's College, Ahmedabad 380 009, India
}

MS received 24 June 2003; revised 5 January 2004

\begin{abstract}
Texturing of two different magnetic fluids were carried out in paraffin wax under the influence of an external magnetic field. The textured samples were characterized using magnetization measurement and a.c. susceptibility techniques. The results are discussed in the light of ratio of anisotropic energy to magnetic and thermal energies.
\end{abstract}

Keywords. Textured ferrofluid; anisotropic energy; a.c. susceptibility.

\section{Introduction}

A colloidal dispersion of nano-sized magnetic particles in a non-magnetic carrier is known as a magnetic fluid. In a magnetic fluid because of rotational freedom of the particles, the magnetization process does not depend upon particle anisotropy. Therefore, the magnetization of the ferrofluid represents the degree of alignment of the magnetic moments of the particles, i.e. Zeeman energy, $\mu H$, where $\mu$ is the magnetic moment of the particle and $H$ the applied magnetic field. For magnetically textured samples (i.e. solidification of matrix in presence of applied magnetic field) orientation of the particle becomes fixed and in such a system in-field magnetization process takes place through the Neel rotation of magnetic moments which are away from the easy axis of particle. So one has to consider the statistical distribution of anisotropy axes at preparation temperature and applied magnetic field. Raikher (1983) calculated the difference between the normalized magnetization curves for magnetically textured samples and ferrofluid considering a mono-disperse system. Recently, Bentivegna et al (1998) modified Raikher's model for a polydispersed system and showed that increase in anisotropy energy (i.e. $K V$, where $K=$ effective anisotropy constant and $V=$ particle volume) is equivalent to 'resistance to magnetization'. They have also shown that the difference between magnetization of fluid and textured fluid is very small when anisotropy energy is much lower than Zeeman energy i.e. $K V \ll \mu H$, at a given temperature. Here, we investigate (i) magnetic properties of two ferrofluids, which differ in magneto crystalline energy of the dispersed particles and (ii) the above fluids are textured in paraffin wax under different texturing magnetic fields. In sample $A$, which contains magnetite

*Author for correspondence particles, the anisotropy energy is much greater than thermal energy at room temperature while in sample B containing $\mathrm{Mn}-\mathrm{Zn}$ ferrite particles, it is the other way. This allows us to study both the limits of Bentivegna et al (1998) theories, i.e. $\mu H \ll K V$ and $\mu H \gg K V$, at a given temperature.

\section{Experimental}

2.1a Preparation of fluid: The ferrite particles of $\mathrm{Fe}_{3} \mathrm{O}_{4}$ (sample A) and that of $\mathrm{Mn}-\mathrm{Zn}$ ferrite (sample B) were prepared using co-precipitation method. The analytical grade reagents were used in proper molar ratio in aqueous solution. The co-precipitation was carried out using sodium hydroxide $(\mathrm{NaOH})$. The details of the preparation conditions for both the samples are reported elsewhere (Upadhyay and Mehta 1993; Upadhyay Trupti et al 1997). These ferrite particles were coated with oleic acid and then dispersed in kerosene. The fluid was centrifuged before mixing with paraffin wax. The homogeneity of the dispersed phase in paraffin wax was confirmed by recording magnetization of different parts of the same sample. The observed variation is $<1 \%$. The structural properties of magnetic particles were studied using X-ray powder diffractometer.

2.1b Texturing of sample: In order to freeze the ferrofluid under different magnetic fields, paraffin wax was used as a solid matrix. Texturing of the fluid was carried out when the paraffin wax was in a molten state. Special care was taken during texturing so that the particles did not aggregate. The texturing of the sample was carried out using an electromagnet having field uniformity within $1 \%$ under different texturing magnetic fields $\left(H_{\mathrm{T}}\right)$. In the present case the samples were prepared under two types of configurations viz. (i) parallel geometry, $H_{\mathrm{T}}(\|)$ (i.e. the 
texturing field keeps the magnetic moments parallel to the measuring field) and (ii) perpendicular geometry, $H_{\mathrm{T}}(\perp)$ (i.e. texturing field keeps the magnetic moments perpendicular to the measuring field).

2.1c Structural characterization: The crystalline structure of the particles was confirmed using Bruker D8
Advance $\mathrm{X}$-ray powder diffractometer $(\mathrm{CuK} \alpha$ radiation, $\lambda=1.5414 \AA$ ). The X-ray patterns were analysed using Rietveld refinement program, which confirms the formation of a single, phase cubic spinel structure. The structural parameters like particle size(diameter), $D_{x}$; lattice parameter, $a$, particle density, $\rho_{\mathrm{p}}$ and cation distribution were obtained using (311) reflection (table 1) (Cullity 1978).

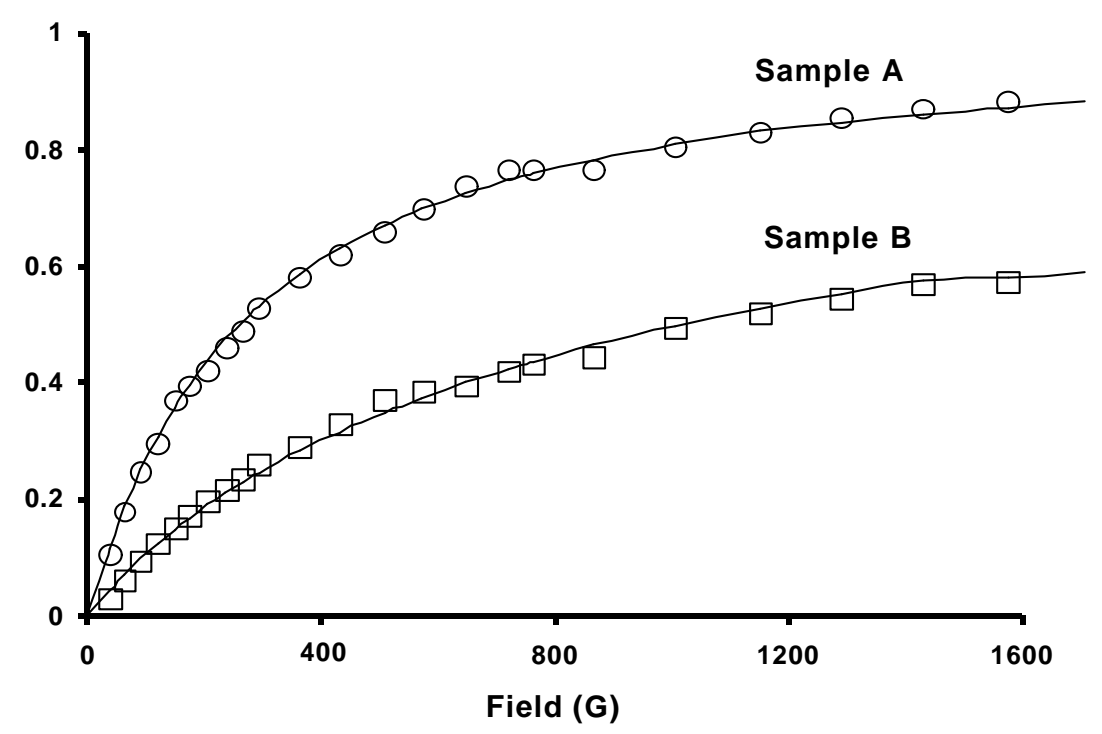

Figure 1. Reduced magnetization vs applied magnetic field for samples A and B at room temperature.

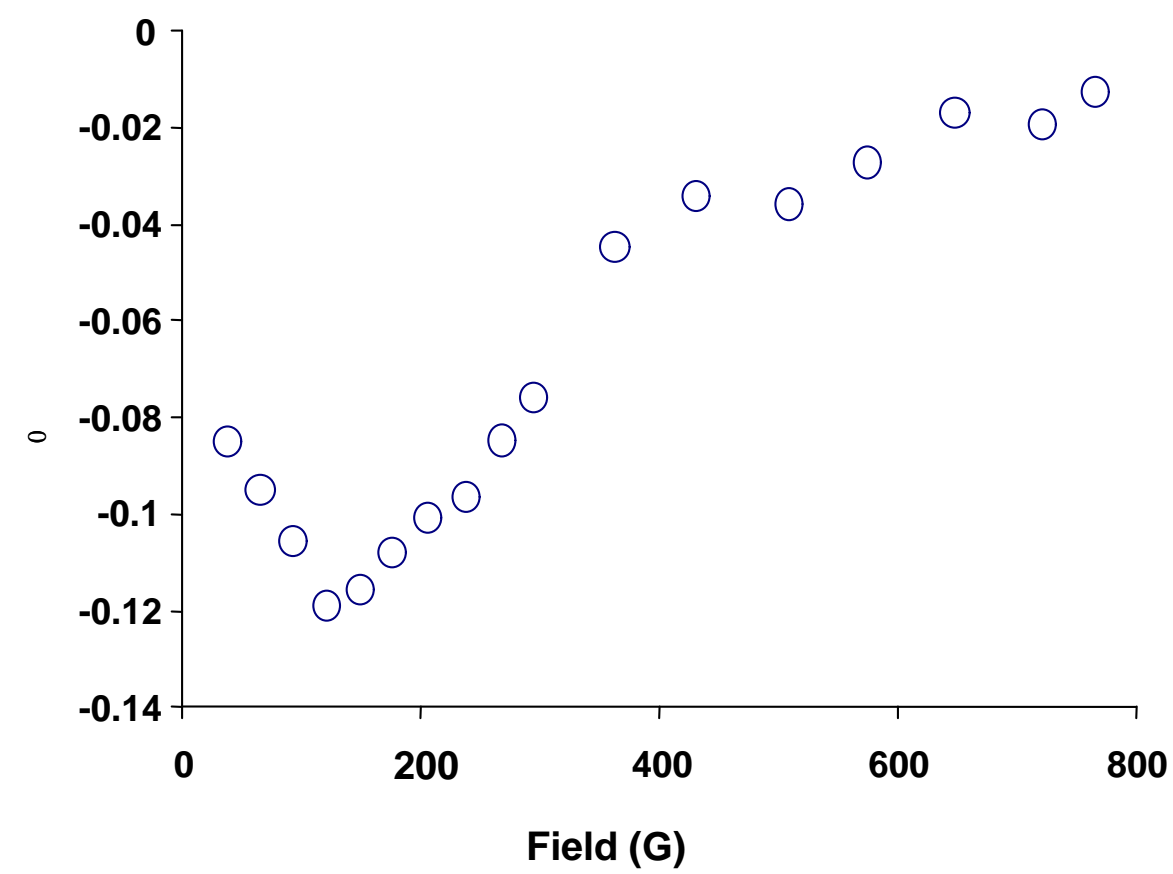

Figure 2. Change in magnetization between fluid and textured samples in zero texturing magnetic field for sample A. 
Table 1. Parameters obtained from magnetization measurement.

\begin{tabular}{lccccc}
\hline Sample & $M_{\mathrm{s}}(\mathrm{G})$ & $M_{\mathrm{d}}(\mathrm{emu} / \mathrm{cc})$ & $D_{\mathrm{m}}(\AA)$ & $D_{\mathrm{x}}(\AA)$ & $\sigma$ \\
\hline $\mathrm{A}\left(\mathrm{Fe}_{3} \mathrm{O}_{4}\right)$ & 220 & 320 & 120 & 150 & $0 \cdot 29$ \\
$\mathrm{~B}(\mathrm{Mn}-\mathrm{Zn}$ ferrite $)$ & 138 & 210 & 71 & 75 & $0 \cdot 31$ \\
\hline
\end{tabular}

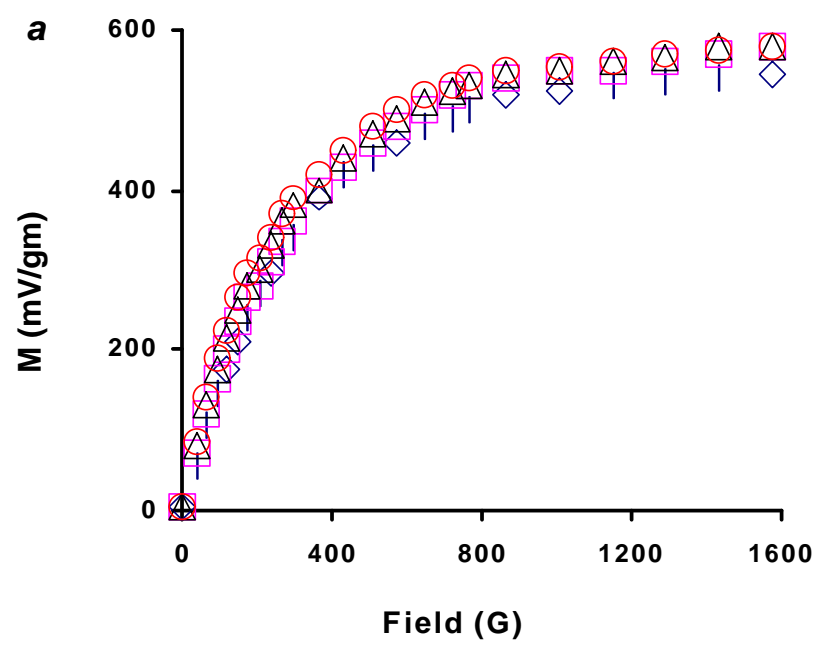

b

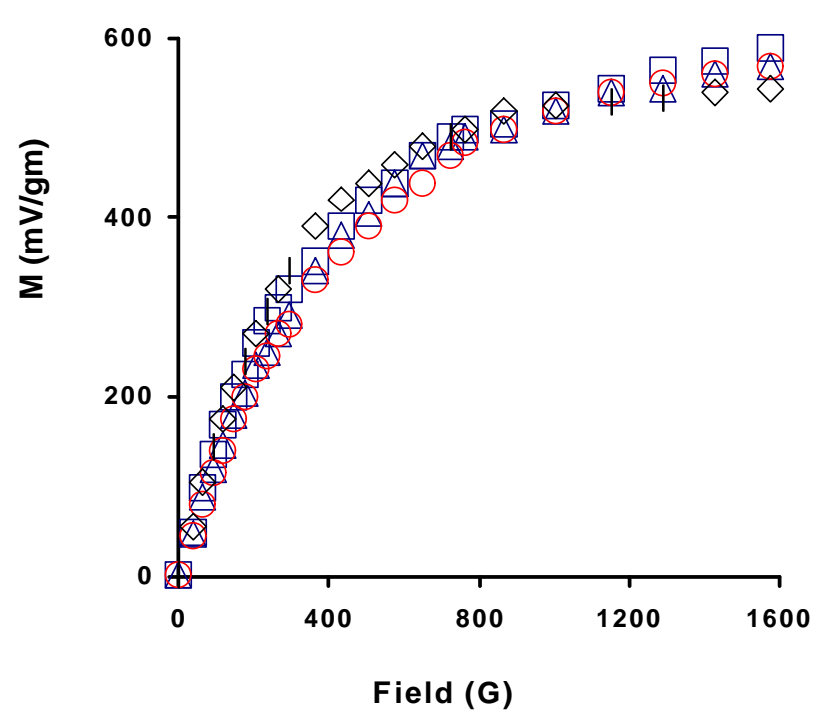

Figure 3. Effect of texturing field on a magnetically textured fluid magnetization for (a) $\left(H_{\mathrm{T}} \| H\right)$ and (b) $\left(H_{\mathrm{T}} \perp H\right)$ for sample A: $(\diamond) H_{\mathrm{T}}=0 \mathrm{G},(\square) H_{\mathrm{T}}=300 \mathrm{G},(\triangle) H_{\mathrm{T}}=600 \mathrm{G},(\mathrm{O}) H_{\mathrm{T}}=1 \mathrm{kG}$.

\section{Results and discussion}

\subsection{Magnetization measurement}

The magnetization measurements were carried out using the search coil method. The induced emf of the coil is measured via storage oscilloscope. The coil is calibrated with the fluids having known magnetization. 3.1a Fluid sample: Figure 1 shows the room temperature magnetization measurements for magnetic fluids, $\mathrm{A}$ and B. Both the systems exhibit zero remanence and coercivity. Usually saturation magnetization of the fluid $\left(M_{\mathrm{s}}\right)$ can be obtained by extrapolating the plot of $M$ (magnetization at a given field, $H$ ) vs $1 / H$ to the infinite field. But in the case where a distribution of particle size is involved (as in the present case), the field required to saturate the smaller particle is very large compared to the bigger particle. Therefore, by extrapolation one may get a wrong value of saturation magnetization. In order to determine the values of $M_{\mathrm{s}}$, we have used Langevin's model incorporating polydispersity of the particle size. According to this, magnetization can be written as,

$$
M=\phi M_{\mathrm{d}} \int_{0}^{\infty} L(\alpha) P(D) \mathrm{d}(D),
$$

where $\phi$ is magnetic volume fraction of the particles and $M_{\mathrm{d}}$ the domain magnetization of particle. Here, we have assumed a log-normal distribution function, $P(D)$, for spherical particles given by

$$
\begin{gathered}
P(D) \mathrm{d} D=\frac{1}{D \sigma(2 \pi)^{1 / 2}} \\
\exp \left[\frac{\ln \left(D / D_{0}\right)^{2}}{2 \sigma^{2}}\right] \mathrm{d} D,
\end{gathered}
$$

where $D$ is the particle diameter, $\sigma$ the standard deviation of $\ln D$ and $\ln D_{\mathrm{m}}$ the mean of $\ln D$. The magnetization curves were fitted using (1) and (2) and appropriate fit parameters were determined (table 1 ).

3.1b Magnetization of frozen samples for $H_{T}=0(Z F C)$ : In magnetic fluid the orientational structure becomes a controllable parameter, therefore, polymerization transforms magnetic fluid into a solid heterogeneous material, the grains of which are mechanically immobilized. Thus solidification fixes the orientational distribution of magnetic particles. If polymerization takes place in absence of magnetic field, the easy axes of magnetic particles are randomly distributed in all directions. Since the system under consideration consists of superparamagnetic particles with a small relaxation time, the existence of texture 
does not influence remanence i.e. $M(0)=0$. When we magnetize this sample there will be a difference between the actual $M(H)$ curve and Langevin's function because, for a frozen system the in-field magnetization process takes place through the Neel rotation of the magnetic moment away from the easy axes of particle direction. Raikher (1983) has shown that an easy way to evaluate the effect of frozen anisotropy axes on the in-field magnetization curve is to plot the difference

$$
\Delta M_{0}=M_{0} / M_{0 \mathrm{~s}}-L(\alpha)
$$

Here $M_{0}$ stands for magnetization of the sample prepared under zero texturing field.

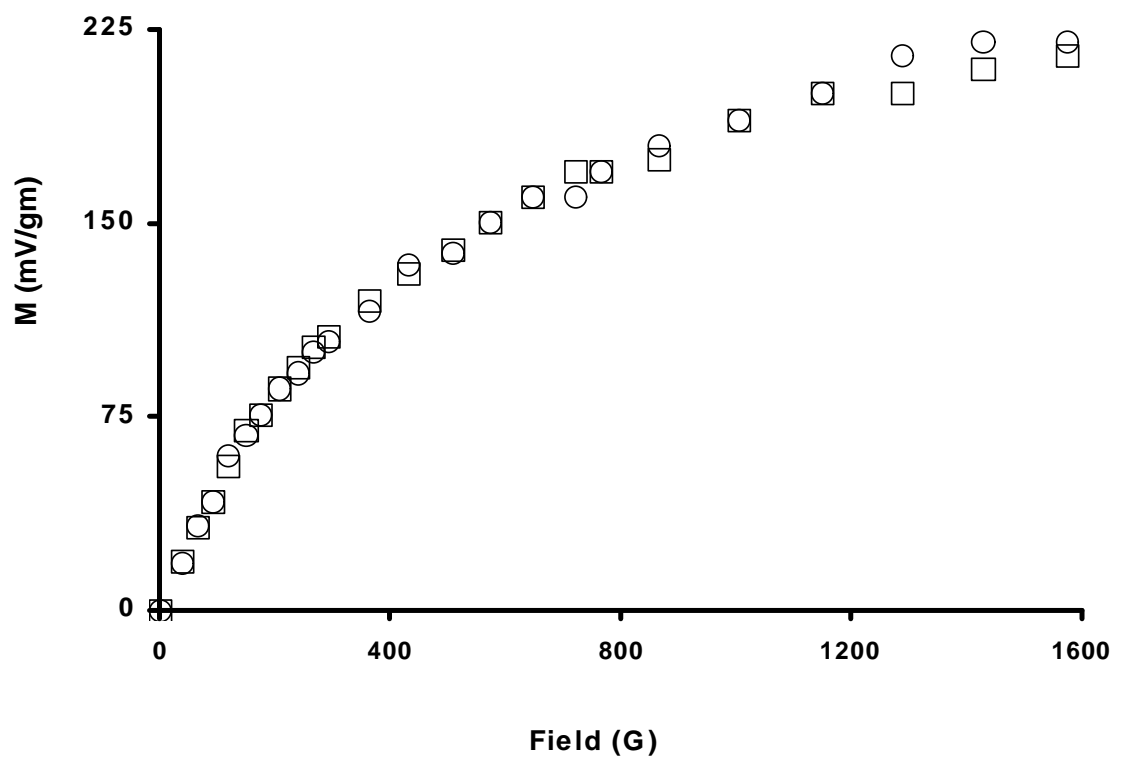

Figure 4. Magnetization measurement for sample $B$ for $1 \mathrm{kG}$ texturing field for $\left(H_{\mathrm{T}} \| H\right)(\square)$ and $\left(H_{\mathrm{T}} \perp H\right)(\mathrm{O})$.

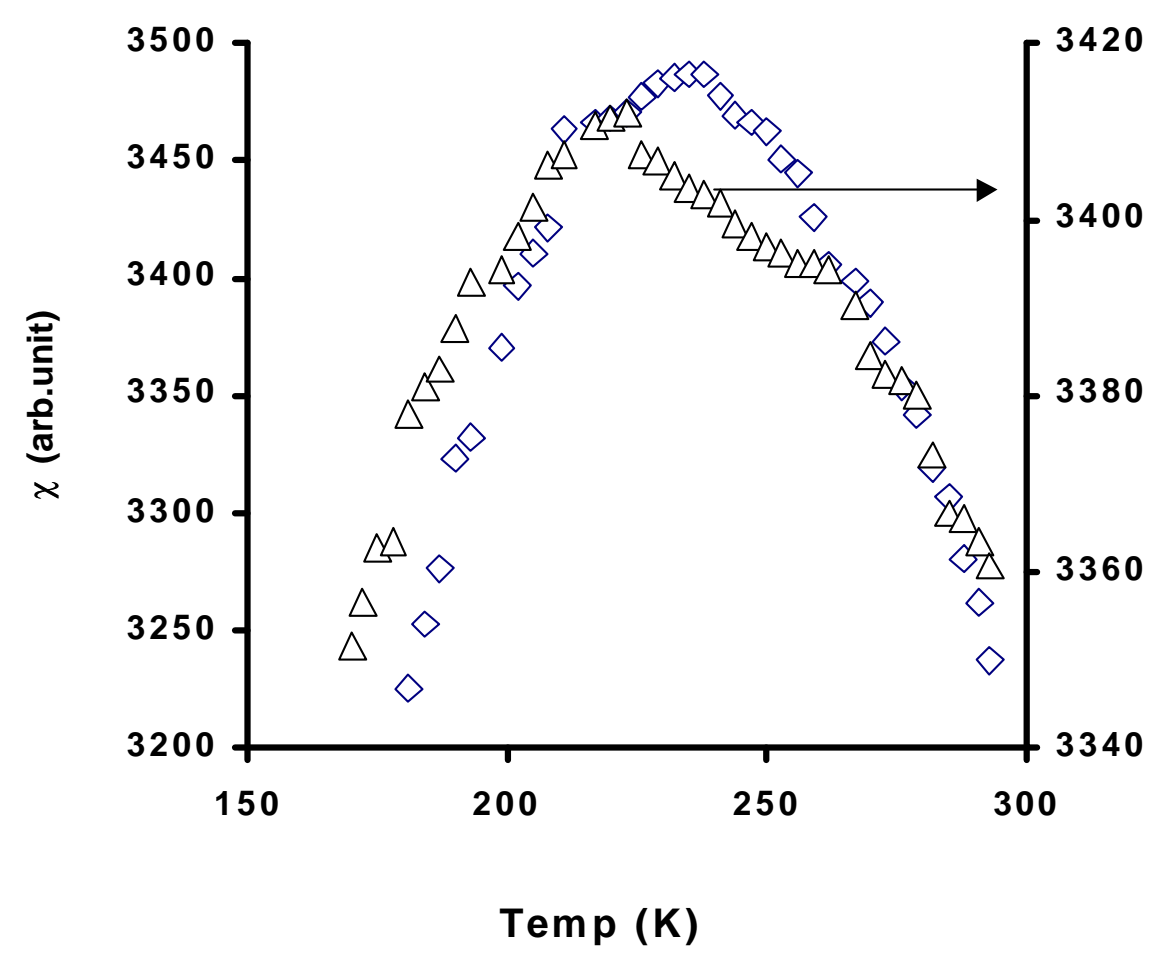

Figure 5. Temperature dependent a.c. susceptibility measurements for sample A: ZFC $(\triangle)$ and FC $(\diamond)$. 


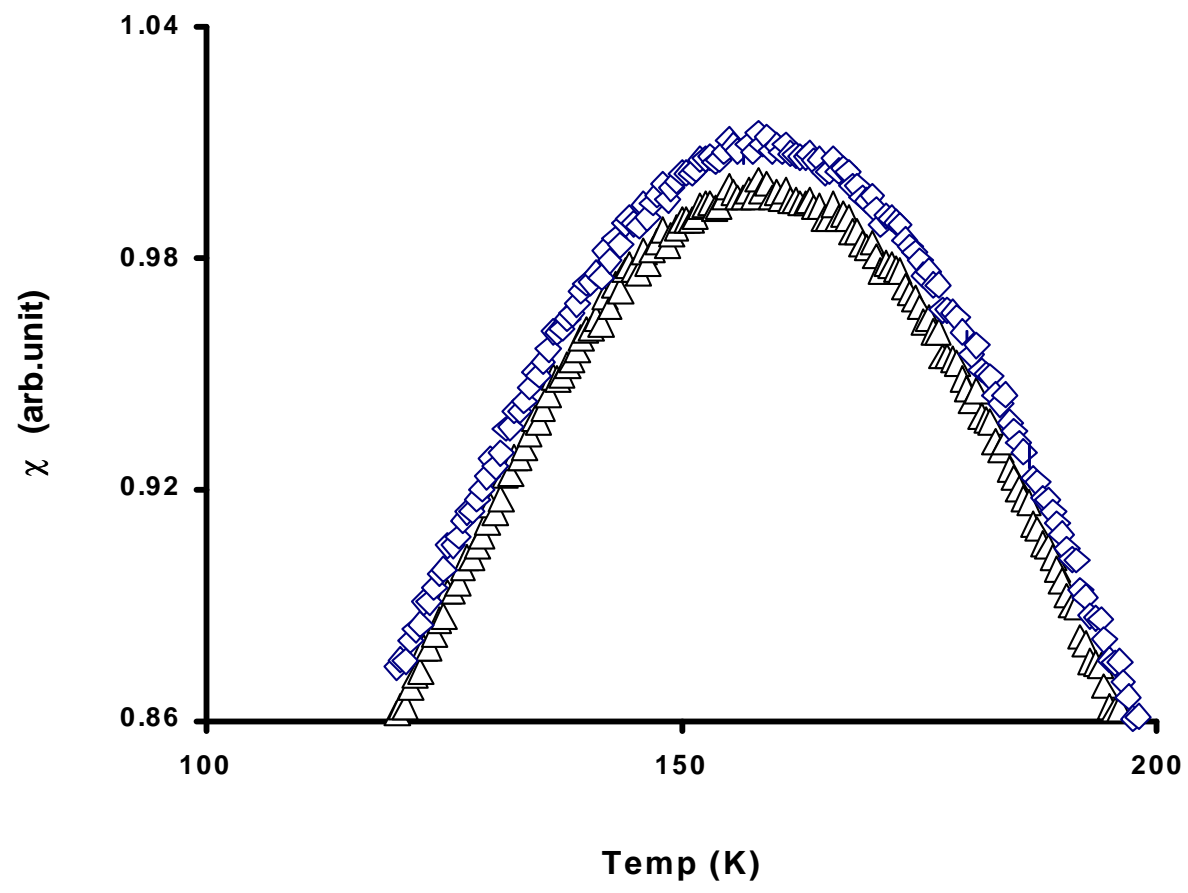

Figure 6. Variation of a.c. susceptibility with temperature for a sample B: $(\triangle) \mathrm{ZFC}$ and $(\diamond) \mathrm{FC}$.

Figure 2 shows the departure of relative magnetization curve of zero field polymerized magnetic particles from Langevin's function for fluid A. The maximum change depends upon the anisotropy energy, $K V$. Therefore, this energy barrier can be considered as a resistance to magnetization. For fluid $\mathrm{B}$, the value of $\Delta M_{0}$ was found to be zero, indicating that in this fluid the anisotropy energy of the particle is negligible with respect to magnetic energy as well as thermal energy i.e. particles may be considered as intrinsic superparamagnetic.

3.1c Magnetization of frozen magnetic sample for $H_{T} \neq 0$ $(F C)$ : In case when the fluids are frozen in the matrix under magnetic field, particles become mechanically immobile due to freezing of the matrix. Two limiting cases may be considered according to the relative orientation of the applied field (i) parallel geometry $\left(H_{\mathrm{T}}(\|)\right)$ and (ii) perpendicular geometry $\left(H_{\mathrm{T}}(\perp)\right)$. Figures $3 \mathrm{a}$ and $\mathrm{b}$ show the magnetization curve of sample A for parallel and perpendicular geometries for different texturing fields. In parallel geometry the alignment of magnetic moment for small induction is favoured by a magnetic texturing field. This effect is more prominent at low field in $M$ vs $H$ curve, because at low field susceptibility is dominated by large particles only.

In figure 4, the in-field magnetization curves for parallel and perpendicular geometry of sample B are shown. In this case, ZFC and FC magnetization curves overlap on each other. This is due to the smaller size of particles and lesser anisotropy value $(K)$ of sample B compared to that of sample $A$ (see table 1). Thus the anisotropy energy is much less than magnetic energy and particles which are aligned in the field direction during texturing come back to their original position as soon as the texturing field is removed. These results support our assumption that the anisotropy energy is much greater than thermal energy for sample A, in case of sample B it is the other way, i.e. $\mu H \ll K V$ and $\mu H \gg K V$ at a given temperature, respectively for samples $\mathrm{A}$ and $\mathrm{B}$.

The above study is carried out in d.c. magnetic field where the time scale of the measurement is of the order of a few seconds. In order to study the effect of a.c. magnetic field on texturing where the time scale is of the order of $10^{-3} \mathrm{~s}$ the a.c. susceptibility measurements were carried out at different temperatures.

\subsection{A.c. susceptibility measurements}

The temperature dependent a.c. susceptibility of both the samples were measured between 77 and $300 \mathrm{~K}$ using indigenously built a.c. susceptometer (Jani et al 2003). The data were recorded for warming cycle. The frequency of measurement for all the samples is kept constant at $211 \mathrm{~Hz}$.

The a.c. susceptibility measures the reversible part of the magnetization curve. In fine magnetic particle system the peak in susceptibility defines the thermally activated spin reversal which is called as blocking temperature, $T_{\mathrm{B}}$, of the particles above which all particles are superparamagnetic. Since superparamagnetism depends on the ratio of $K V / k T$ for a given field strength, therefore, one must observe this effect in samples A and B also. 
Figure 5 shows the effect of texturing field on the susceptibility curve of sample A for ZFC sample $\left(H_{\mathrm{T}}=0 \mathrm{G}\right)$ and FC sample $\left(H_{\mathrm{T}} \| 1 \mathrm{kG}\right.$, i.e. the texturing field is directed parallel to the a.c. magnetic field), respectively. In figure 5 three discontinuities are observed at temperatures around $195 \mathrm{~K}, 230 \mathrm{~K}$ and $263 \mathrm{~K}$. These discontinuities may arise because of the stress, induced by the cooling of paraffin wax. Similar discontinuities were also observed in FC sample, but the peak at low temperature i.e. $195 \mathrm{~K}$ of ZFC sample is shifted towards higher temperature $(209 \mathrm{~K})$ while $263 \mathrm{~K}$ peak shifts towards lower temperature $(243 \mathrm{~K})$ resulting in a slight shift in a central peak. This shift in a peak is related to the applied field strength. During the solidification the probability of forming a cluster increases because of the larger size of the particle in sample A. Moreover, the magnitude of susceptibility for FC sample increases four times compared to that of ZFC sample. This may be due to the effect of structure induced in the system while texturing. Similar behaviour is also observed by Jeyadevan and Nakatsuka (1995) for FC and ZFC fluid samples.

Figure 6 shows the susceptibility curve for sample B. In this case no discontinuity was observed. Further, the peak position remains unaltered in ZFC and FC samples. This shows that sample B behaves as superparamagnetic even at $80 \mathrm{~K}$. So the magnetic moment of the particle can relax through Neel relaxation mechanism at that temperature and field.

\section{Conclusions}

Texturing of two different samples having $\mu H \gg K V$ and $\mu H \ll K V$ at a given temperature are carried out in paraf- fin wax. The magnetization and a.c. susceptibility measurement of field cooled and zero field cooled samples are studied. For sample A, the magnetization measurement shows different values for ZFC and FC while it remains identical for sample $B$. The results of a.c. susceptibility for sample A shows three discontinuities in $\mathrm{ZFC}$ as well as FC samples and these peaks are shifted in FC compared to ZFC samples, while no discontinuities are observed for sample B and the blocking temperature remains identical for FC and ZFC samples.

\section{Acknowledgements}

This work was carried out under DST project No. DST$\mathrm{M}-15$. One of the authors (MG) is thankful to the Principal, St. Xavier's College, Ahmedabad, for encouragement.

\section{References}

Bentivegna F et al 1998 J. Appl. Phys. 837776

Cullity B D 1978 Elements of X-ray diffraction (Notre Dame, Indiana: A.W. Publishing Co.) 2nd ed.

Jani K H, Upadhyay R V and Mehta R V 2003 Proceedings of recent advances in nanotechnology of magnetic fluids (New Delhi: NPL) p. 211

Jeyadevan B and Nakatsuka K 1995 J. Magn. Magn. Mater. 14960

Raikher Yu L 1983 J. Magn. Magn. Mater. 3911

Upadhyay R V and Mehta R V 1993 Pramana-J. Phys. 41 429

Upadhyay Trupti, Upadhyay R V, Mehta R V, Goyal P S and Aswal V K 1997 Phys. Rev. B55 5585 